\title{
Research on Application of New 10kV High Voltage Electric Energy Meter Based on All-fiber Optical Current Transformer
}

\author{
Fuli Yang ${ }^{1}$, Lujun Zhang ${ }^{2}$ and Bin $\mathrm{Li}^{2}$ \\ ${ }^{1}$ Electric Power Research Institute of Chongqing Electric Power Company of State Grid, Chongqing, China \\ ${ }^{2}$ Chongqing Qianwei Jibao Power Equipment Co., Ltd., Chongqing, China
}

\begin{abstract}
This paper introduces application of all-fiber optical current transformer to $10 \mathrm{kV}$ high voltage electric energy meter, proposes new application of $10 \mathrm{kV}$ current sampling by all-fiber optical current transformer, analyzes technical advantages of new $10 \mathrm{kV}$ high voltage electric energy meter and makes scheme verification based on tests of new $10 \mathrm{kV}$ high voltage electric energy meter.
\end{abstract}

Keywords-10kV; all-fiber optical current transformer; high voltage electric energy meter

\section{Traditional High Voltage Metering Device}

High voltage metering device is a metering device that connects the parties of power generation, supply and utilization and is used for trade settlement and is widely used, so there are high requirements for its safety, reliability and accuracy. Currently, for $10 \mathrm{kV}$ high voltage electric energy meters in China, the method of combining "traditional electromagnetic transformer" with "multifunction watt-hour meter" is mainly used. Main problems of this traditional high voltage metering device are that: a) system composition error of the whole metering device is produced by transformer, electric energy meter, connecting wire resistance and contact resistance and overall metering error cannot be determined uniquely. $b$ ) It is hard to ensure safety and there are faults and hidden dangers such as magnetic saturation, ferromagnetic resonance and easy combustion and explosion. c) Operation energy consumption is high and high voltage electromagnetic transformers consume a lot of resources such as copper, iron and insulation materials. d) Stealing electricity at low voltage side easily occurs due to many external connecting lines of metering circuits; facilities for preventing stealing electricity and manpower need to be provided additionally. e) Electromagnetic transformer can only output analogy quantity and have narrow frequency band and poor linearity and cannot adapt development trend of power system digitization and intelligence[1].

So current $10 \mathrm{kV}$ high voltage metering device shall be improved by innovative design[2].

\section{High Voltage Electric EnERGy Meter}

High voltage electric energy meter breaks through inherent technical solution of traditional high voltage metering device, uses electronic transformer for sensing and sampling, directly outputs $\mathrm{mV}$ and $\mathrm{mA}$ weak signals required by energy metering unit, reduces conversion between signals of standard voltage and current, reduces output capacity, realizes energy metering at high voltage side and sends data to display and remote units at low voltage side in the optical fiber or wireless manner.

High voltage electric energy meter constituted by the above scheme is an integrated direct metering device at high voltage side and uses new principle and structure design so that it overcomes many shortcomings of traditional high voltage metering device and has the advantages such as safety and reliability, accurate metering, energy and material saving, prevention of stealing electricity, and easy installation[3].

Operation situation shows that high voltage electric energy meter used for $10 \mathrm{kV}$ distribution network can safely and reliably substitute traditional high voltage metering device, save resources and energy, be consistent with national industrial policies and have powerful vitality.

Currently, technical route of high voltage electric energy meter developed in China is classified into two categories:

\section{A. Weak Output Voltage Transformer + Weak Output Current Transformer + Energy Metering Unit}

When weak output transformers are used, secondary voltage output is reduced within $12 \mathrm{~V}$ from $100 \mathrm{~V}$ or $100 / \sqrt{3} \mathrm{~V}$, current is reduced to $0.1 \mathrm{~A}$ from $5 \mathrm{~A}$ or $1 \mathrm{~A}$ and output capacity is reduced to few volt-amperes. As their volumes and weights are reduced, they can be packaged in a box together with energy metering unit to form an integrated metering device.

Advantages of the scheme: compared with traditional power transformer, it significantly saves raw materials and has small power consumption and loss, small size, light weight, convenient installation and maintenance on site, high sensitivity, big dynamic range and good stability.

Disadvantages of the scheme: as it will not be saturated within big change range of voltage and current, probability of burning secondary winding greatly increases in the case of overvoltage and overcurrent; with the emerging of new electronic transformer, its advantages of size and weight are no longer in existence.

\section{B. Electronic Transformer + Energy Metering Unit}

Since the 1960s, with the development of electronic, digital and communication technologies, new electronic transformers have been developed based on different metering principles to overcome shortcomings of traditional transformers such as 
high energy consumption, much material consumption and unsatisfactory safety and to bring challenges to traditional transformers.

Electronic transformers mainly include resistance voltage divider, capacitive voltage divider, Rogowski current transformer and other mixed type electronic transformers or optical transformers[4]. Compared with traditional electromagnetic transformers, electronic transformers have no core and have simple and reliable insulation structure, light weight and good linearity and they are free of ferromagnetic resonance and saturation and can output digital quantity. Electronic Voltage Transformer GB/T20840.7 and Electronic Current Transformer GB/T20840.8 were issued and implemented in China in 2007. Considering voltage grade, cost, structure, stability and other factors, mixed type electronic transformers are mainly used for high voltage electric energy meters for $10 \mathrm{kV}$ distribution network[5]. The electronic transformers have the above advantages, but voltage dividers will be affected by stability, distributed capacitance, temperature change, voltage to ground and other factors and Rogowski current transformers are also affected by temperature and external magnetic field and have shortcomings such as measuring dead zone existing under small current and output characteristic affected by coil winding and way of integration[6].

Therefore, high voltage electric energy meter based on new principle of current sampling can be explored on the basis of electronic high voltage electric energy meter developed successfully at present.

\section{New 10KV High Voltage Electric ENERGy Meter BASED ON ALL-FIBER OPTICAL CURRENT TRANSFORMER}

To overcome shortcomings of current transformers for current high voltage electric energy meters, in this paper we propose one new high voltage electric energy meter using all-fiber optical current transformer, with the structure of all-fiber optical current transformer + current method voltage transformer + energy metering unit.

\section{A. All-fiber Optical Current Transformer}

All-fiber optical current transformer is based on Faraday magneto-optical effect. The light emitted by light source passes through the coupler and then polarizes by the polarizer, passes through the modulator for phase modulation and is divided into two perpendicular beams at the polarization direction and passes through conductive fiber and then enters into sensor fiber and the two beams are subject to coherence stack at photoelectric detector at the end of the sensor fiber.

When there is no current in primary conductor, the both beams have the same relative propagation velocity, namely there is no phase difference upon coherence stack at the detector; when a current is applied, propagation velocities of the both beams will have relative change, namely there will be phase difference and superimposed light intensity at the detector will change. Current size of corresponding primary conductor can be measured through measurement of light intensity. As shown in Figure I.
Using high birefraction keeping oval fiber and current closed-loop feedback correction method, all-fiber optical current transformer described in this paper thoroughly solves the problems of anti-vibrating and impact, scale production, temperature compensation, long-term stability and small signal measurement existing in the industry and has many advantages such as good insulating property and electromagnetic compatibility, fast response speed, wide measurement frequency band, big dynamic range, and low-carbon and environmental protection.

\section{B. Voltage Transducer of Current Method}

The current method shall is used for the voltage sampling of the new $10 \mathrm{kV}$ high voltage energy meter, as shown in figure II: phase A: the resistance R1, after connected in series with the input end of micro current transducer T4, is connected with both ends of the measured voltage. The secondary side input end of micro current transducer T4 is connected in parallel with resistance $\mathrm{R} 4$, of which a segment is connected to the electricity metering unit U1 via potentiometer RP1. So do phase $B$ and phase $C$.

The output end and input end of micro current transducers T4-T6 are subject to high/low voltage isolation. The units of high/low voltage isolation can be the isolation devices of photoelectric type, fiber type, electromagnetic type, electrical insulation type, oil isolation type or solid isolation type. The resistance R4-R6can not only be the resistance load, but also capacitance load, inductive load or the composition. The micro current transducers T4-T6 can be the photoelectric micro current transducer, fiber micro current transducer, electromagnetic micro current transducer and traversing micro current transducer as well. The error between the measured voltage $\mathrm{V}$ and secondary voltage $\mathrm{v}$ is made less than the required value within $80 \%, 100 \%$ and $120 \%$ ranges via the compensation regulation and the linearity shall also meet the accuracy requirement. The voltage is input into the electricity metering unit U1 as the standard signal of high-voltage energy measurement, and the ratio of transformation of $\mathrm{V} / \mathrm{v}$ is multiplied into the electricity metering unit U1.

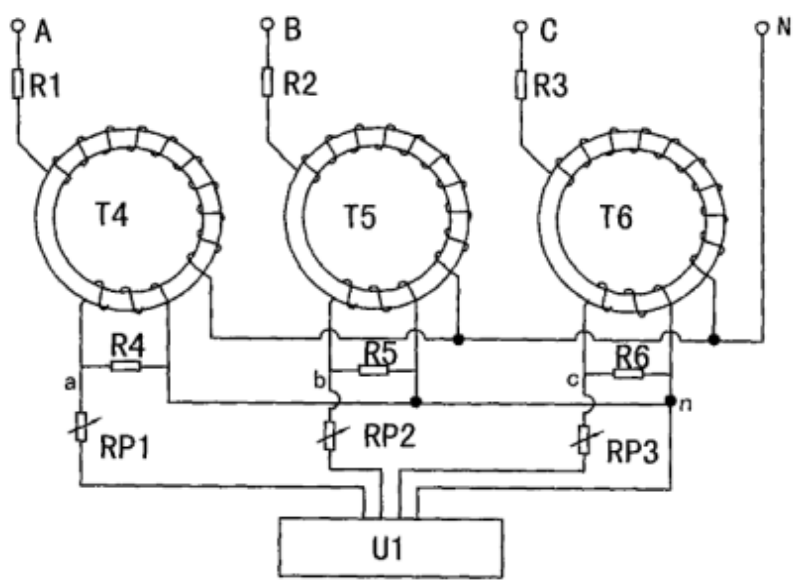

FIGURE I. SCHEMATIC DIAGRAM OF VOLTAGE TRANSDUCER OF CURRENT METHOD 


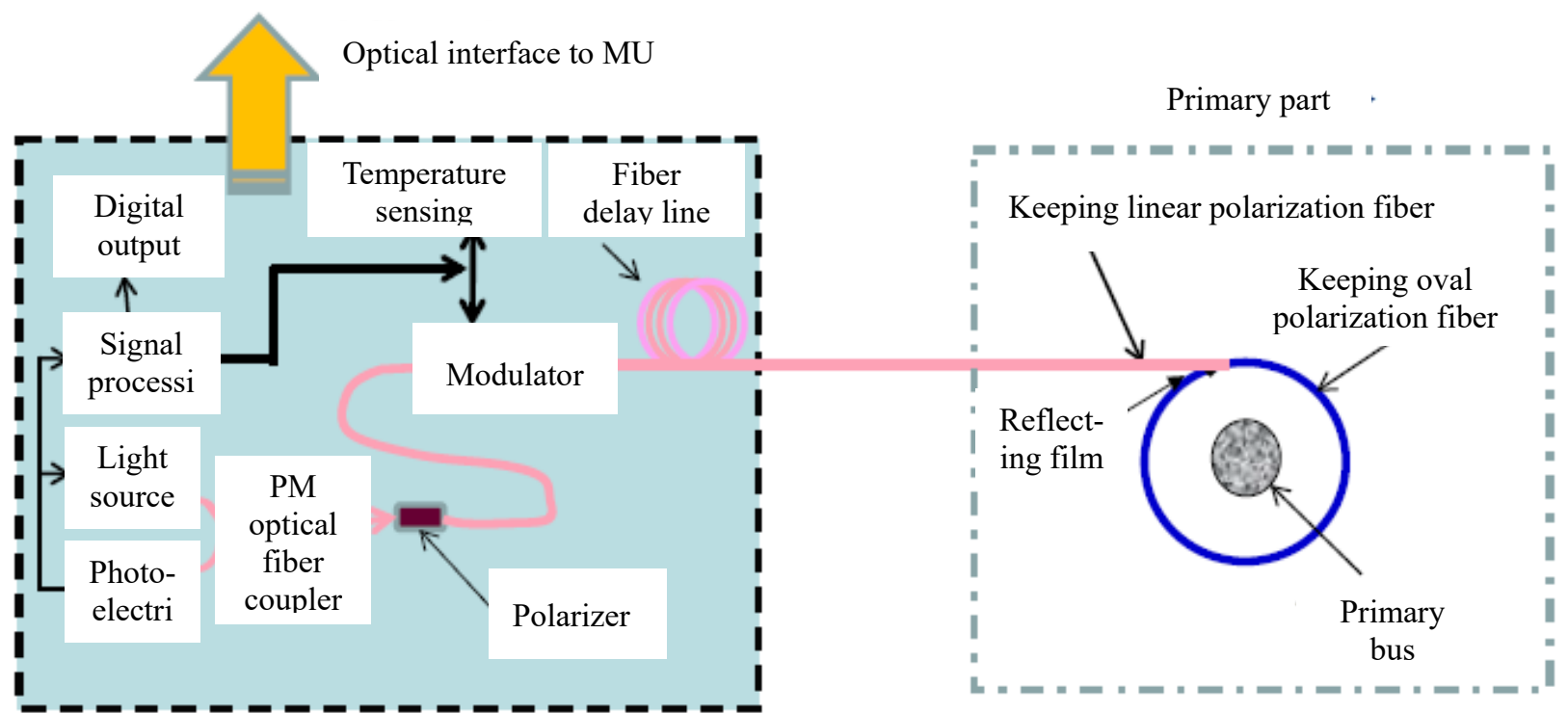

Optoelectronic signal processing unit

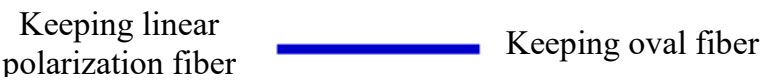

FIGURE II. PRINCIPAL Block DiAgram OF All-FIBER OPTICAL CURRENT TranSFormer

The current method is used to measure the high voltage, which solves many security problems caused by electromagnetic resonance, higher harmonic and switching over-voltage existed in the electromagnetic transformer, avoids the problem that the measurement error and deviation in the power system of different topological structures varies due to the use of voltage divider affected by distributed capacitance to ground and meets the requirements of measuring and monitoring.

\section{Electricity Metering Unit}

The scheme of special measurement chip+ MCU is used for the new $10 \mathrm{kV}$ high voltage energy meter. The conditioning and sampling units are integrated in the special measurement chip and the data processing algorithm is consolidated so as to directly work out the final energy data; the MCU directly reads the measurement result of measurement chip or performs the simple data processing so as to realize the measurement function of electric energy meter. The scheme meets the design concept of modularization, makes the basic measurement and storage capability of communication separated in logic and can make both realize separate uplevel or change but not mutually influenced.

\section{TEST VALIDATION}

\section{A. Accuracy Test}

The standard unit of high voltage electric energy meter that is qualified by measurement standard is used to test measuring accuracy of new $10 \mathrm{kV}$ high voltage electric energy meter, with measuring accuracy of level 0.1 , it can also be used to entirely check level 0.5 Shigh voltage electric energy meter and test overall metering error of traditional HV metering device.
The new $10 \mathrm{kV}$ high voltage electric energy meter to be checked has a rated voltage of $10 \mathrm{kV}$, a rated current of $100 \mathrm{~A}$, and active pulse constant of $640 \mathrm{imp} / \mathrm{MWh}$, the measured vale of positive active energy error is shown in Table I, with its accuracy level up to $0.5 \mathrm{~S}$.

\section{TABLE I. ACCURACY TEST OF COMPLETE SET}

\begin{tabular}{|c|c|c|c|c|}
\hline \multicolumn{2}{|c|}{} & $\begin{array}{c}\text { Phase A } \\
\mathbf{( \% )}\end{array}$ & Phase C (\%) & $\begin{array}{c}\text { Combined } \\
\text { phase(\%) }\end{array}$ \\
\hline \multirow{5}{*}{$\mathbf{P F = 1}$} & $\mathbf{1 2 0 A}$ & -0.15 & 0.35 & 0.30 \\
\cline { 2 - 5 } & $\mathbf{1 0 0 A}$ & -0.05 & 0.20 & 0.25 \\
\cline { 2 - 5 } & $\mathbf{5 0 A}$ & $/$ & $/$ & 0.10 \\
\cline { 2 - 5 } & $\mathbf{1 0 A}$ & $/$ & $/$ & 0.00 \\
\cline { 2 - 5 } & $\mathbf{5 A}$ & -0.25 & 0.30 & -0.20 \\
\cline { 2 - 5 } & $\mathbf{1 A}$ & $/$ & $/$ & -0.15 \\
\hline \multirow{5}{*}{$\mathbf{P F = 0 . 5 L}$} & $\mathbf{1 2 0 A}$ & 0.10 & 0.30 & 0.30 \\
\cline { 2 - 5 } & $\mathbf{1 0 0 A}$ & 0.20 & 0.10 & 0.20 \\
\cline { 2 - 5 } & $\mathbf{5 0 A}$ & $/$ & $/$ & -0.20 \\
\cline { 2 - 5 } & $\mathbf{2 0 A}$ & $/$ & $/$ & 0.20 \\
\cline { 2 - 5 } & $\mathbf{1 0 A}$ & -0.45 & 0.45 & 0.25 \\
\cline { 2 - 5 } & $\mathbf{2 A}$ & $/$ & $/$ & 0.15 \\
\hline & $\mathbf{1 2 0 A}$ & & & 0.20 \\
\cline { 2 - 5 } & $\mathbf{1 0 0 A}$ & & & 0.15 \\
\cline { 2 - 5 } $\mathbf{P F = 0 . 8 C}$ & $\mathbf{5 0 A}$ & $/$ & $/$ & 0.15 \\
\cline { 2 - 5 } & $\mathbf{2 0 A}$ & $/$ & $/$ & -0.25 \\
\cline { 2 - 5 } & $\mathbf{1 0 A}$ & & & 0.10 \\
\cline { 2 - 5 } & $\mathbf{2 A}$ & $/$ & $/$ & \\
\hline
\end{tabular}

\section{B. Test of Insulation Performance}

The tests of isolation performance in the draft for approval of General Technical Requirements of High Voltage Electric Energy Meter: 


\section{1) Test of AC Withstand Voltage}

Technical requirements: the power frequency withstand voltage of $30 \mathrm{kV}$ and $42 \mathrm{kV}$ shall be forced between phases and between phase and ground respectively for 1 minute, if there's no electric discharge phenomenon in the tested objects such as breakdown or flashover, then the test is passed.

\section{2) Test of Lightning Impulse Withstand Voltage}

Technical requirements: the standard lightning impulse voltage shall be the bi-exponent pulse with $1.2 \mu$ s of time to crest $\mathrm{T} 1$ and $50 \mu \mathrm{s}$ of time of half peak value T2, 5 times of normal polarity lightning impulse withstand voltage (peak value) shall be forced between the phases and between phase and ground, if there's no electric discharge phenomenon in the tested objects such as breakdown or flashover, then the test is passed.

\section{3) Test of Partial Discharge}

Technical requirements: the voltage of $14.4 \mathrm{kV}$ and $8.3 \mathrm{kV}$ shall be forced between the phases and between phase and ground respectively and the magnitude of partial discharge shall be less than $20 \mathrm{pF}$.

The new $10 \mathrm{kV}$ high voltage electric energy meter passed the above tests of isolation performance in the Chongqing Electric Power Research Institute.

\section{CONCLUSION}

The new $10 \mathrm{kV}$ high voltage electric energy meter based on all-fiber optical current transformer has the advantages of compact structure, small volume, light weight, usage of solid insulation and simplification of manufacturing process, meanwhile, it can meet the technical requirements of high voltage measurement products in the indicator aspects of measurement accuracy and isolation performance, which makes the beneficial attempt for the all-fiber optical current transformer in the $10 \mathrm{kV}$ power distribution network, thus the network operation is to be conducted for validation of reliability and stability.

\section{REFERENCES}

[1] Xin Liu, Beige Yang, Jian Wang, Fuying Li. Study on New Type High Voltage Electric Energy Meter[J]. Automation of Electric Power Systems, 2004, 28 (9).

[2] Shun Hu, Zhigui Xu. Development of High Voltage Electric Energy Meter[J]. National Productivity Promotion Center of Electrical Instrumentation, Industry Conference.

[3] Xiongying Duan. Theory and Experiment of Electronic Power Transformer: [Doctoral Dissertation]. Wuhan: Huazhong University of Science and Technology, 2002.

[4] Qiufeng Shang. Study on the Practical Method of Optical Current Transformer: [Doctoral Dissertation]. Baoding: North China Electric Power University, 2004.

[5] Yanbing Liu, Hongbin Li et al. Principle, Technology and Application of Electronic Transformer [M]. Science Press

[6] Chaoyang Zhang, Chunxi Zhang et al. Calculation and Test of Phase Difference of All-fiber Optical Current Transformer [J]. Chinese Journal of Scientific Instrument. 2009, 30(1). 